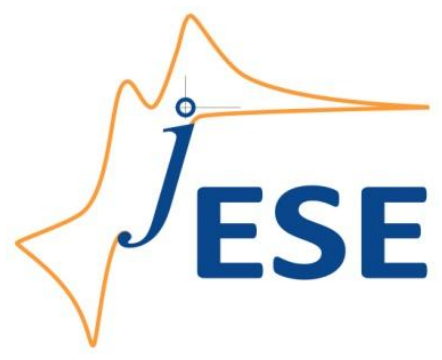

Open Access : : ISSN 1847-9286

www.jESE-online.org

Original scientific paper

\title{
Development of an electrochemical sensor for the determina- tion of the total antioxidant capacity in berries based on boron doped diamond
}

\author{
BRUNA PEKEC ${ }^{* * * * *}$, BIRGIT FEKETEFÖLDI**, VOLKER RIBITSCH**, ASTRID \\ ORTNER*** and KURT KALCHER* ${ }^{*}$ \\ *Institute of Chemistry, Department of Analytical Chemistry, Karl-Franzens University, Graz, Austria \\ **Joanneum Research Forschungsgesellschaft mbH - Materials, Weiz, Austria \\ ***Institute of Pharmaceutical Science, Department of Pharmaceutical Chemistry, Karl-Franzens \\ University, Graz, Austria \\ ${ }^{\square}$ Corresponding Author: E-mail: kurt.kalcher@uni-graz.at, Stremayrgasse 16/III, 8010; Tel.: +43 (0)316 380 - \\ 5310; 5313; Fax: +43 (0)316 380-9845
}

Received: August 28, 2012; Revised: October 18, 2012; Published: November 06, 2012

\begin{abstract}
Many antioxidants can be electrochemically oxidized using graphite-based electrodes; nevertheless problems arise due to the strong adsorption of redox species at the sensing area. We have demonstrated that boron doped diamond (BDD) electrodes do not show this property, which can be exploited for the design of a new amperometric sensor for the quantification of antioxidants as "total antioxidant capacity" (AOC). As reference substances hydroquinone (HQ) and 6-hydroxy-2,5,7,8-tetramethylchromane-2-carboxylic acid (Trolox) were studied in more detail. The supporting electrolyte was a phosphate buffer solution (PBS, $0.1 \mathrm{~mol} / \mathrm{L}, \mathrm{pH} 7.0)$. The limits of detection (LOD) were $1.5 \mathrm{mg} / \mathrm{L}$ and $2.5 \mathrm{mg} / \mathrm{L}$ for $H Q$ and Trolox, respectively. The repeatability was $3 \% R S D$ for concentration of $200 \mathrm{mg} / \mathrm{L} \mathrm{HQ}$. The method could be applied for the determination of $A O C$ in different berry samples, such as strawberry, blueberry, grape and bramble. A comparison with a standard photometric assay showed good correlation between both methods. The BDD sensor features good reproducibility without fatiguing over at least two months of operation.
\end{abstract}

\section{Keywords}

Boron doped diamond electrode; total antioxidant capacity; amperometric sensor; berry extracts. 


\section{Introduction}

The term "antioxidant" identifies a huge number of substances, in majority of cases phenolic or polyphenolic substances that inhibit the oxidation of molecules. They are strongly reducing agents that neutralize reactive oxygen species (ROS). These ROS are oxidizing radicals or molecules that are formed during the respiratory chain and cause damage on cell own material. Antioxidants neutralize these species and therefore they are of enormous scientific interest.

The free scavenger effect of antioxidants has been in use for many years to prolong the stability of lipid foods especially of edible oils. Reports go back to 1947 where butylated hydroxyanisole was used as the first antioxidant for the prevention of the deterioration of fat-containing food [1-2]. Nowadays antioxidants are also in the medicinal focus because of their tissue protecting effects by neutralization of ROS. Therapeutic progress has been made with many diseases that show a high degeneration rate, such are diabetes, coronary heart failure or Alzheimer disease [3-5]. Ever since in 2003 Sinclair et al. found out that resveratrol, an antioxidant occurring in grape, extends the lifespan of yeast cells and causes a decrease of typical aging effects, antioxidants became also interesting for cosmetic branches and for anti-aging research [67].

Antioxidants are mostly secondary plant metabolites. Especially berries, such as grape, red or black currant, bramble or raspberries, are known to form high amounts of flavonoids. Their synthesis is a response of the plant to stress attacks like strong light exposure or fungal or microbial infection. In the latter case, the plant uses them as phytoalexines for tissue protection. Unfortunately, isolated antioxidants are highly sensitive to light, temperature and oxygen. Although natural antioxidants are an interesting tool to prevent lipid products, e.g. oily food or cosmetics, to become rancid, their stabilization is still a problem to be solved.

Responding to a growing interest some analytical methods, mostly photometric assays, were developed. Standardized techniques are the ORAC-assay (oxygen radical absorbance capacity) involving hydrogen transfer reactions, the Folin-Ciocalteu-assay and the TEAC-assay (Trolox equivalent antioxidant capacity) that base on electron transfer reactions [8]. Although these methods have been well approved they are rather complex, time-consuming and use high priced chemicals or test kits.

Most antioxidants show electrochemical activity and could be determined using voltammetric or amperometric methods. Unfortunately, during the electrochemical process reduced and oxidized species adsorb very strongly on the surface of many electrode types, such as graphite or carbon paste electrodes (CPE).

Diamond electrodes offer an alternative approach. Ever since in 1987 Pleskov et al. [9] commenced studies on the electrochemistry of boron doped diamond (BDD) their development and fundamental research gained rising tendency. In contrast to graphite electrodes the C-sp ${ }^{3}$ hybridization and tetrahedral bonding of boron doped diamond electrodes (BDDE) leads to properties like chemical inertness, hardness, thermal conductivity and low fatigue [10]. Their main field of application is the treatment of wastewater [11]. The potential window of BDDEs is much wider $(-0.75$ to $+2.35 \mathrm{~V})$ than of graphite electrodes $(-0.5$ to $+1 \mathrm{~V})$ because neither oxygen nor hydrogen evolution interfere in the analysis. Because commercial BDDEs are usually synthesized by chemical vapor deposition (CVD) they contain non-diamond and metallic impurities [10]. Also, surface oxygen can cause disturbances and it needs to be removed. For surface cleaning and pretreatment of BDDEs a few suggestions, like acid washing or anodic polarization, have been offered [12,13]. 
In this work the pretreatment of boron doped diamond electrodes by polarization in $\mathrm{Na}_{2} \mathrm{SO}_{4}$ at $+1 \mathrm{~V}$, and the use of this electrode for the electrochemical determination of antioxidants in ethanolic berry extract, was investigated.

\section{Experimental}

\section{Chemicals and materials}

The investigated target analytes were phenolic and polyphenolic ingredients of different berries (grape, bramble, blueberry, strawberry) that are known to show antioxidative potential [14]. Therefore ferulic acid, vanillic acid, syringic acid, coumaric acid, sinapic acid, caffeic acid and $p$ hydroxybenzoic acid, all purchased from Sigma-Aldrich GmbH (Vienna, Austria), were selected. Trolox [( \pm )-6-Hydroxy-2,5,7,8-tetramethylchromane-2-carboxylic acid] and hydroquinone (HQ) were chosen as reference substances and were purchased, like all other standards, from SigmaAldrich $\mathrm{GmbH}$ (Vienna, Austria). To prepare the standard solutions the appropriate amount of substance was dissolved in deionized water. The aqueous solutions were prepared freshly before use, kept cool and protected from light. Phosphate buffer $\left(\mathrm{Na}_{2} \mathrm{HPO}_{4} / \mathrm{NaH}_{2} \mathrm{PO}_{4} 0.1 \mathrm{~mol} / \mathrm{L}\right.$, $\mathrm{pH}$ 7) was selected as electrolyte medium.

\section{Apparatus}

All electrochemical experiments were performed using potentiostat (Metrohm AUTOLAB ${ }^{\circledR}$ PGstat302N) in a three electrode arrangement controlled by the corresponding software (NOVA 1.6). The BDDEs purchased from Fraunhofer (Michigan, USA), plated on titanium substrate with gold as a contact, a saturated calomel electrode (SCE) and a platinum wire were used as working, reference and auxiliary electrode respectively. All potential values in this work are given against SCE.

Cyclic voltammetry and hydrodynamic amperometry were chosen as techniques to obtain the electrochemical data. In the case of cyclic voltammetry the explored potential window was between $-1 \mathrm{~V}$ and $+1.2 \mathrm{~V}$. The other operating parameters were: scan rate: $50.0 \mathrm{mV} / \mathrm{s}$, step potential: $2.0 \mathrm{mV}$ and time interval: $0.050 \mathrm{~s}$. For hydrodynamic amperometry measurements the characteristic potential of the oxidation current peak was selected.

\section{Measuring procedure}

The BDDE was conditioned at $+1 \mathrm{~V}$ for $1 \mathrm{~h}$ in $0.1 \mathrm{~mol} / \mathrm{L} \mathrm{Na} \mathrm{SO}_{4}$ solution prior to the start and also between the measurements to remove impurities and for the baseline correction.

All experiments were carried out in $20 \mathrm{~mL}$ phosphate buffer $(0.1 \mathrm{M}, \mathrm{pH}$ 7) after degassing with argon. Aliquots of the standard solutions were added to the cell and cyclic voltammograms and hydrodynamic amperograms, respectively, were recorded by applying the aforementioned parameters.

\section{Photometric analysis}

The photometric Trolox Equivalent Antioxidant Capacity assay (TEAC-Assay) was selected as a reference method. According to the report of Li et al. [15] the redox indicator 2,2'-Azinobis-(3-ethylbenzthiazolin-6-sulfonic acid) (ABTS) forms a radical in contact with potassium persulfate. The ABTS radical cation is of green color which decreases in intensity upon addition of substances with antioxidative potential. The solution was diluted using phosphate buffer saline $(\mathrm{pH}=7)$ until it showed an absorption of $0.700 \pm 0.050$ at $734 \mathrm{~nm}$. To $3 \mathrm{~mL}$ of that solution $100 \mu \mathrm{L}$ of the sample were added and the absorption decrease was measured. For the calibration Trolox and HQ 
solutions of $0.1,0.2,0.3$ and $0.4 \mathrm{~mol} / \mathrm{L}$ were used and the $A O C$ of the berry samples was calculated as Trolox- or hydroquinone-equivalent (TE, HQE).

\section{Preparation of the samples}

The berry marcs (donated from Grünewald Fruchtsaft $\mathrm{GmbH}$ ) were dried at $50{ }^{\circ} \mathrm{C}$. For the recovery of the phenolic compounds the milled raw material was extracted with a mixture of absolute ethanol and water $(75: 25 \mathrm{v} / \mathrm{v})$ at a 8:1 solvent-to-sample ratio (volume to mass of ovendried marc). The extraction was performed using sonication at a temperature of $50{ }^{\circ} \mathrm{C}$ for $15 \mathrm{~min}$. After centrifugation $(7000 \mathrm{rpm}, 20 \mathrm{~min}$ ) the extract was then filtered through a glass fiber filter (type AP2029325, Millipore) and stored at $4{ }^{\circ} \mathrm{C}$.

\section{Results and Discussion}

\section{Cyclic voltammetry}

Cyclic voltammetry (CV) was basically used to determine the oxidation potential of the standard berry antioxidants with CPEs and BDDEs (Table 1). The quoted potentials correspond to the maximum of the oxidation current registered in the anodic scan. Typical CVs of hydroquinone and Trolox are shown in Fig. 1. As can be seen the electrochemical behavior of both substances on both electrodes is rather similar with the exception of carbon paste electrode where the currents are significantly higher. This behavior is due to the adsorption of the analyte at CPE and therefore due to the strong $\pi$ electron interaction of the aromatic ring with the graphite structure. Both substances were used as reference compounds, by converting the response of other antioxidants to Trolox- (TE) and hydroquinone-equivalents (HQE), respectively.

a

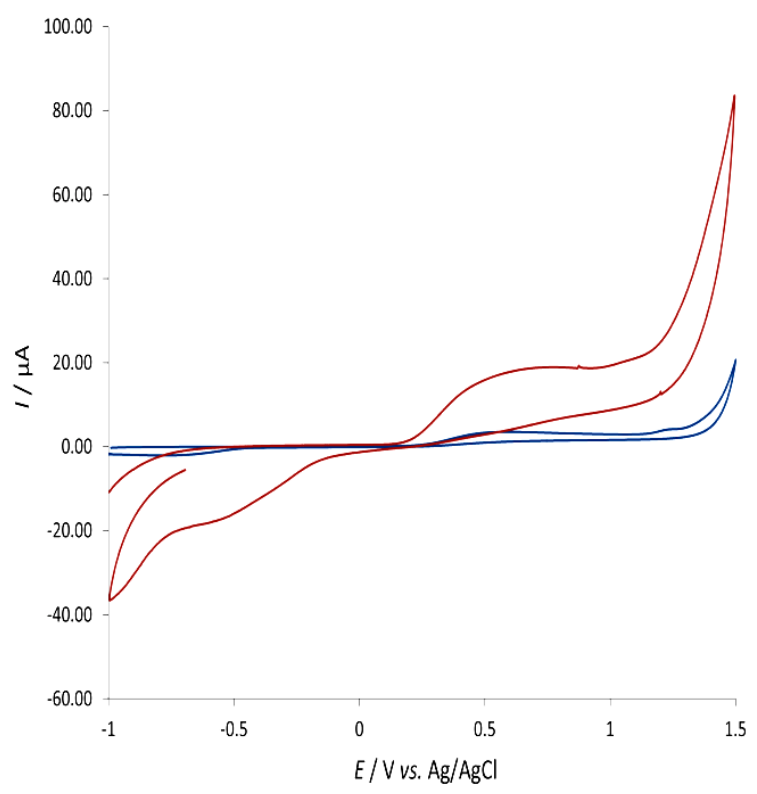

b

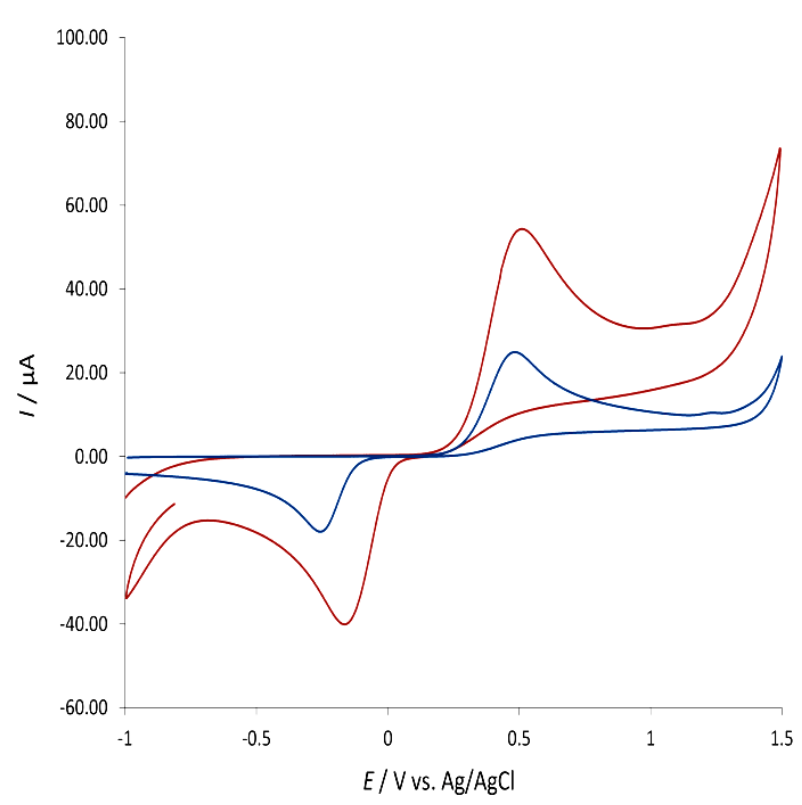

Figure 1. Cyclic voltammograms of $30 \mathrm{mg} / \mathrm{L}$ (a) Trolox and (b) HQ measured in PBS (0.1 mol/L, $\mathrm{pH} 7)$ on $B D D E$ and $\cdot C P E$.

The investigated antioxidants (Table 1) produce typical peak currents in CV at BDDEs in the range of 4 to $8 \mathrm{~mA} \mathrm{~mol}^{-1}$ with the exception of $\mathrm{HQ}$ which is about four times higher; probably because $\mathrm{HQ}$ ideally interacts with the graphite surface finally yielding benzoquinone, whereas 
other oxidants are reduced to the corresponding R-O- radical with ensuing follow-up effects. Again it is evident that carbon paste electrode favors adsorption of the oxidation product which exerts a positive effect for the first cycle in cyclic voltammetry but it is deteriorating the signals in further scans and in amperometry (see below) by blocking the active surface area.

Since the sensors based on boron-doped diamond should be used for alcoholic extracts of berries, the influence of ethanol on the signal was investigated. Concentrations from 0.5 to $20 \%$ ethanol were added to the supporting electrolyte; no influence on peak position or peak height was observed.

Table 1. Oxidation potentials, $E_{p a,}$ and peak currents, $i_{p a}$ from cyclic voltammetry of typical antioxidants found in berries. Concentration: 30, respectively $90 \mathrm{mg} / \mathrm{L}$, supporting electrolyte: $P B S(0.1 \mathrm{~mol} / \mathrm{L}, \mathrm{pH} 7)$; potential window: -1.5 to $+1.5 \mathrm{~V}$, scan rate: $50 \mathrm{mV} / \mathrm{s}$.

\begin{tabular}{|c|c|c|c|c|c|}
\hline \multirow{2}{*}{ Substance } & \multirow{2}{*}{ Formula } & \multicolumn{2}{|c|}{ CPE } & \multicolumn{2}{|c|}{ BDDE } \\
\hline & & $E_{\mathrm{pa}} / \mathrm{V}$ & $i_{\mathrm{pa}} / \mathrm{mA} \mathrm{mol}^{-1}$ & $E_{\mathrm{pa}} / \mathrm{V}$ & $i_{\mathrm{pa}} / \mathrm{mA} \mathrm{mol}^{-1}$ \\
\hline Syringic acid & & 0.95 & 23.3 & 0.80 & 8.2 \\
\hline Sinapic acid & & 0.70 & 15.2 & 0.65 & 4.3 \\
\hline Caffeic acid & & 0.48 & 10.2 & 0.50 & 7.5 \\
\hline Vanillic acid & & 0.88 & 78.6 & 0.88 & 4.9 \\
\hline Ferulic acid & & 0.50 & 19.2 & 0.50 & 7.9 \\
\hline Coumaric acid & & 0.90 & 37.5 & 0.75 & 5.1 \\
\hline p-Hydroxybenzoic acid & & 0.90 & 148.5 & 1.0 & 6.9 \\
\hline Trolox & & 0.50 & 14.2 & 0.50 & 6.2 \\
\hline Ascorbic acid & & 0.61 & 56.4 & 0.54 & 36.4 \\
\hline Hydroquinone & & 0.50 & 44.0 & 0.50 & 26.7 \\
\hline
\end{tabular}




\section{Hydrodynamic amperometry}

\section{Basic studies}

Hydrodynamic amperometry was applied to the antioxidants listed in Table 1 applying a working potential which corresponds to the maximum of the oxidation current in CV. After attaining a stable baseline the analytes were added to a stirred solution.

Figure 2 shows a comparison of the electrochemical behavior of HQ between BDDE (a) and $\mathrm{CPE}$ (b) electrodes under identical conditions. As can be seen a strong adsorption of the quinone on the CPE leads to a fast decrease of the signal due to blocking of the active electrode surface area with a very small current steps after ensuing additions. BDDE does not show any obvious adsorption therefore, after addition of the electrochemically active analyte, the signal remains constant. Repetitive additions lead to clear step-like increases of current. For this reason BDDE seems to be an ideal electrode material for the determination of phenolic antioxidants due to the absent tendency for adsorption.
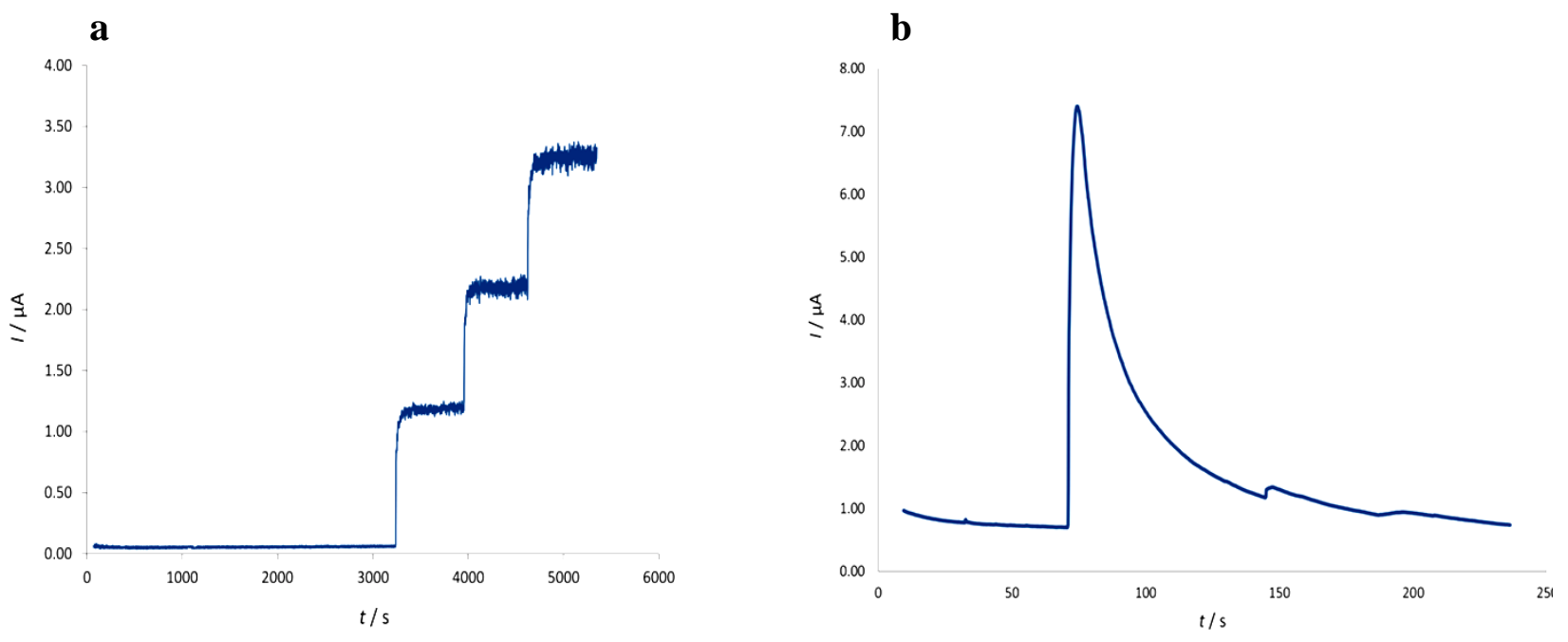

Figure 2. Hydrodynamic amperograms of subsequent additions of $30 \mathrm{mg} / \mathrm{L} \mathrm{HQ}$ in phosphate buffer $(0.1 \mathrm{M}$, $p H$ 7) using (a) BDDE and (b) CPE. Working potential: $+1 \mathrm{~V}$.

\section{Validation of analytical parameters}

The analytical method based on hydrodynamic amperometry was evaluated with hydroquinone and Trolox as standards. Under optimized conditions the linearity range of the BDD-sensor was estimated to be between 10 and $400 \mathrm{mg} / \mathrm{L}$ for Trolox and 10 to $300 \mathrm{mg} / \mathrm{L}$ for HQ (Fig. 3). The calibration curves can be described by the following regression equations:

$$
\begin{aligned}
& i_{\text {HYDROQUINONE }} / \mu \mathrm{A}=43.04 \times \gamma /(\mathrm{mg} / \mathrm{L})+0.387 \text { and } \\
& i_{\text {TROLOX }} / \mu \mathrm{A}=22.09 \times \gamma /(\mathrm{mg} / \mathrm{L})+0.609
\end{aligned}
$$

both with a mean correlation coefficient of $R=0.999$. For the concentrations above $400 \mathrm{mg} / \mathrm{L}$ (Trolox) and $300 \mathrm{mg} / \mathrm{L}$ (HQ) the calibration graph levels off but still remains linear to concentrations above $1 \mathrm{~g} / \mathrm{L}$, in fact with a very small sensitivity. The limit of detection (LOD) and the limit of quantification (LOQ estimated as the three-fold of LOD) were determined as $1.5 \mathrm{mg} / \mathrm{L}$ and $4.5 \mathrm{mg} / \mathrm{L}$ for $\mathrm{HQ}$, and $2.5 \mathrm{mg} / \mathrm{L}$ and $7.5 \mathrm{mg} / \mathrm{L}$ for Trolox according to the International Conference on Harmonisation, ICH, Quality Guidelines for Analytical Evaluation [Q2(R1)] guidelines for visual evaluation [16]. 
The precision experiments $(100,200,375,625,750$ and $875 \mathrm{mg} / \mathrm{L} ; \mathrm{n}=4$ ) showed a relative standard deviation (RSD) of 3-8 \% and a RSD of the slope of $3 \%$. The intercept of the calibration curves ranged from around $0.4(\mathrm{HQ})$ to $0.6 \mu \mathrm{A}$ (Trolox) with a RSD of about $5 \%$ in different calibrations $(n=4)$.

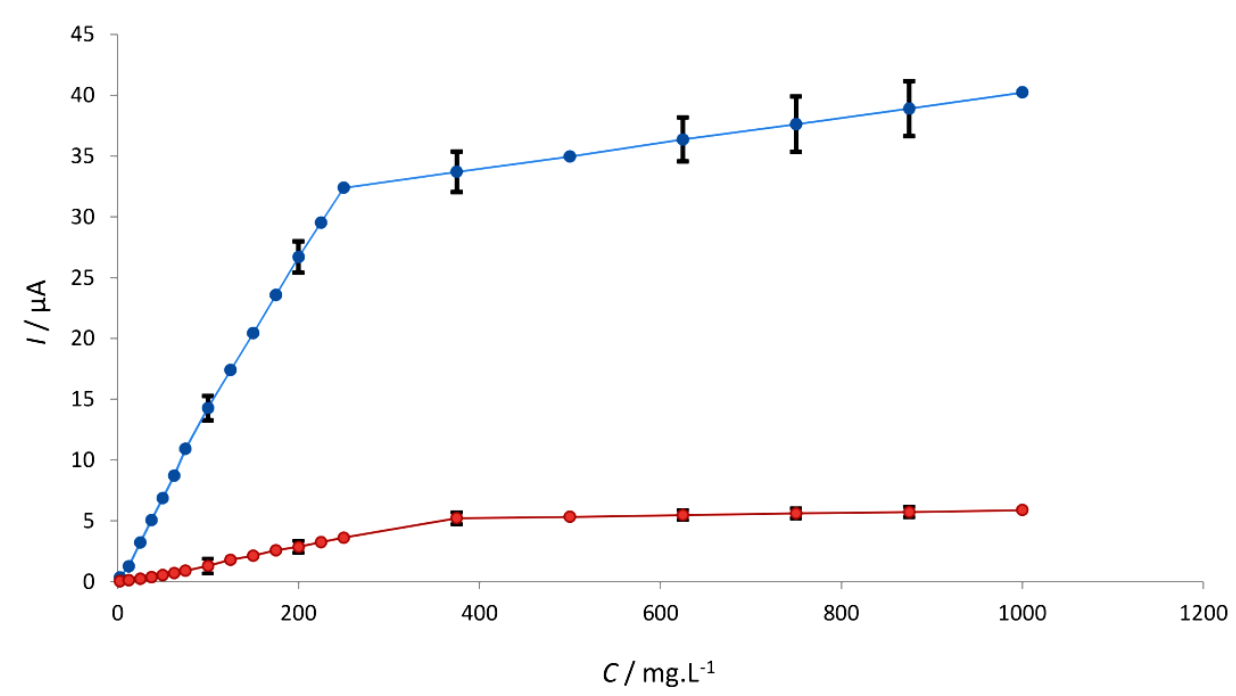

Figure 3. Calibration curve of the BDD-sensor $10-1200 \mathrm{mg} / \mathrm{L}$ concentration range of $\bullet \mathrm{HQ}$ and $\bullet$ Trolox.

Application of the sensor in samples

After it was demonstrated that the BDDEs showed only insignificant adsorption effects to the redox products of the investigated antioxidants they were applied to chosen samples. Thus, ethanolic extracts were prepared using red grape, bramble, blueberry and strawberry. The extracts were injected directly to the analytical cell without further dissolution or preparation. 20 $\mathrm{mL}$ of phosphate bufferwas transferred into the electrochemical cell and after the baseline stabilization, $100 \mu \mathrm{L}$ of the extract was injected. The extracts were analyzed using hydrodynamic amperometry at $+1 \mathrm{~V}$ and the AOC was estimated as Trolox equivalent (TE) or hydroquinone equivalent (HQE) using the corresponding calibration curves. The capacities of the berry samples were determined in the range of 8 and $23 \mathrm{mM}$ TE/HQE (Table 2) and are in a good agreement with the results of the photometric reference method.

Table 2. Comparison of the electrochemical and photometric total antioxidant capacity measurements of berry extracts.

\begin{tabular}{lcccc}
\hline & \multicolumn{2}{c}{ Trolox equivalent, mmol/L } & \multicolumn{2}{c}{ Hydroquinone equivalent, mmol/L } \\
& TEAC Assay & Electrochemical BDD sensor & TEAC Assay & Electrochemical BDD sensor \\
\hline Grape & $23.4 \pm 1.2$ & $23.9 \pm 1.2$ & $22.6 \pm 1.1$ & $23.3 \pm 1.2$ \\
Bramble & $12.4 \pm 0.6$ & $12.4 \pm 0.6$ & $12.1 \pm 0.6$ & $13.4 \pm 0.7$ \\
Blueberry & $18.5 \pm 0.9$ & $18.1 \pm 0.9$ & $18.4 \pm 0.9$ & $17.9 \pm 0.8$ \\
Strawberry & $8.9 \pm 0.4$ & $8.9 \pm 0.4$ & $8.8 \pm 0.4$ & $9.1 \pm 0.5$ \\
\hline
\end{tabular}

Figure 4 shows a comparison between electrochemical signals determined using the BDD sensor and the decrease of absorption $(\Delta A)$ measured using the TEAC-Assay. It can be seen clearly that there is a directly proportional relation between the absorption signal decrease and 
amperometric currents. The method employing electrochemical sensors seems well applicable to detect the antioxidant capacity of ethanolic berry extracts.

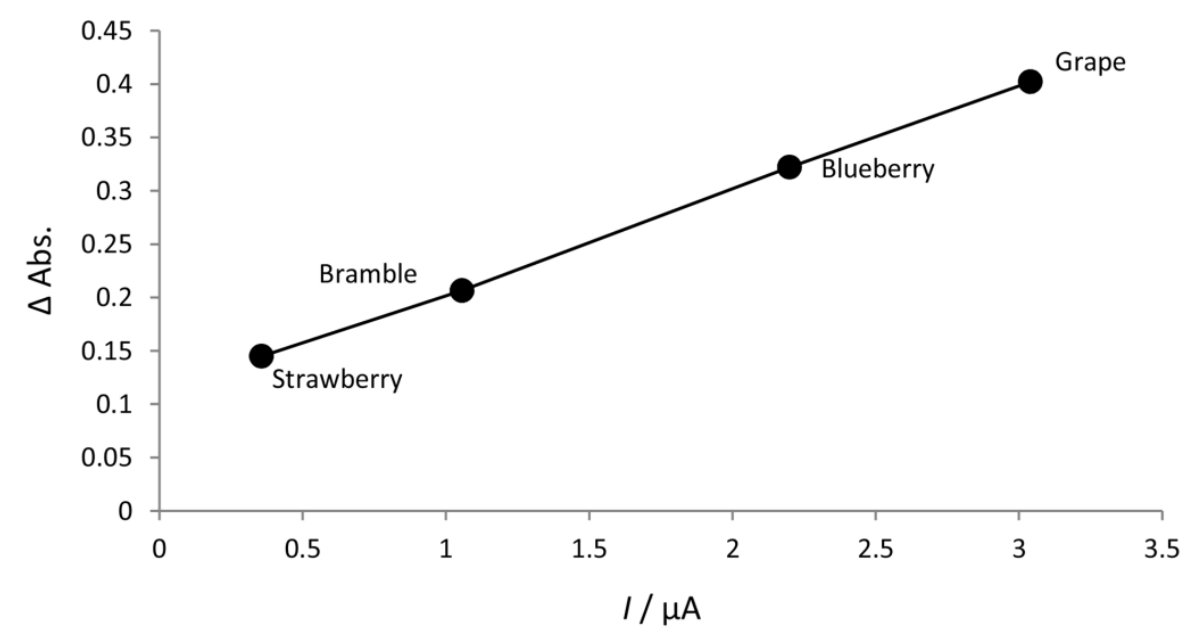

Figure 4. Comparison of electrochemical data recorded in current intensity and photometric results charted as decrease of absorption.

Our studies show that the evaluated Trolox and hydroquinone equivalents from amperometric measurements with BDDEs are correlating well with the optical reference method (Table 2). This was also verified with individual antioxidants (Table 1) which produce the same antioxidant equivalent capacities with both procedures, amperometric and optical.

Maybe, in complex samples, substances which can be oxidized at an operational potential of 1.0 $V$ could be present and do not contribute to the antioxidant capacity. In fact, we could not, so far, observe such a behavior with ethanolic extracts of berries.

\section{Conclusions}

The investigations show that boron doped diamond used as working electrode in electrochemical analytics is an attractive tool because of its many advantages. Due to its low feature for the adsorption of redox products it can be used for quantification of antioxidants even in complex material. The designed sensor is inexpensive because it can be used for repetitive measurements, easy to prepare, and with high reproducibility .

Acknowledgements: The financial support of the Österreichische ForschungsförderungsgeselIschaft (FFG) for project "Antiflavo" (No.827334) is highly acknowledged.

\section{References}

[1] E.R. Sherwin, J. Am. Oil Chem. Soc. 55 (1978) 809-814

[2] E.N. Frankel, Food Chem. 57 (1996) 51-55

[3] H. Kaneto, Y. Miyagawa, T. Matsuoka, Y. Fujitani, Y. Umayahara, T. Hanafusa, Y. Matsuzawa, Y. Yamasaki, M. Hori, Diabetes 48 (1999) 2398-2406

[4] K.L. Hamilton, J.L. Staib, T. Phillips, S.L. Lennon, S.K. Powers, Free Radical Biol. Med. 34 (2003) 800-809

[5] M.J. Engelhart, M.I. Geerings, A. Ruitenberg, J.C. van Swieten, A. Hofman, J.C.M. Wittermann, M.M.B. Breteler, J. Amer. Med. Assoc. 287 (2002) 3223-3229

[6] K.T. Howitz, K.J. Bittermann, H.Y. Cohen, D.W. Lemming, S. Lavu, J.G. Wood, R.E. Zipkin, P. Chung, Kisielewski, L.L. Zhang, B. Scherer, D.A. Sinclaire, Nature 425 (2003) 191-196 
[7] D. Valenzano, E. Terzibasi, T. Genade, A. Cattaneo, L. Domenici, A. Cellerino, Curr. Biol. 16 (2006) 296-300

[8] R.L. Prior, X. Wu, K. Schaich, J. Agr. Food Chem. 53 (2005) 4290-4302

[9] Y.V. Pleskov, A.Y. Sakharova, M.D. Krotova, L.L. Bouilov, B.V. Spitsyn, J. Electroanal. Chem. 228 (1987) 19-27

[10] J.H.T. Luong, K.B. Male, J.D. Glennon, Analyst 134 (2009) 1965-1979

[11] M.A.Q. Alfaro, S. Ferro, C.A. Martinez-Huitle, Y.M. Vong, J. Brazil. Chem. Soc. 17 (2006) 227236

[12] M.C. Granger, M. Witek, J. Xu, J. Wang, M. Hupert, A. Hanks, M.D. Koppang, J.E. Butler, G. Lucazeou, M. Mermoux, J.W. Strojek, G.M. Swain, Anal. Chem. 72 (2000) 3793-3804

[13] H.B. Suffredini, V.A. Pedrosa, L. Codognoto, S.A.S. Machado, R.C. Rocha-Filho, L.A. Avaca, Electrochim. Acta 49 (2004) 4021-4026

[14] M. Monagas, B. Bartolomé, C. Gómez-Cordovés, Food Sci. Nutr. 45 (2005) 85-118

[15] H.B. Li, K.W. Cheng, C.C. Wong, K.W. Fan, F.Chen, Y. Jiang, Food Chem. 102 (2007) 771-776

[16] Validation of analytical procedures - Methodology, Federal Register 62 (1997) 27463 